Quim. Nova, Vol. 35, No. 5, 1036-1040, 2012

\title{
DESENVOLVIMENTO E VALIDAÇÃO DE UM MÉTODO ANALÍTICO POR CLAE PARA QUANTIFICAÇÃO DE ÁCIDO URSÓLICO EM DISPERSÕ̃ES SÓLIDAS
}

\author{
Josimar de Oliveira Eloy, Erika Cristina Vargas de Oliveira, Samantha Sant'Anna Marotta-Oliveira, Juliana Saraiva e \\ Juliana Maldonado Marchetti*
}

Faculdade de Ciências Farmacêuticas de Ribeirão Preto, Universidade de São Paulo, Av. do Café, s/n, 14040-903 Ribeirão Preto - SP, Brasil

Recebido em 18/8/11; aceito em 22/11/11; publicado na web em 20/1/12

\begin{abstract}
DEVELOPMENT AND VALIDATION OF A HPLC METHOD FOR QUANTIFICATION OF URSOLIC ACID IN SOLID DISPERSIONS. Ursolic acid is a natural molecule that presents several pharmacological properties. In this work, an analytical method by RP-HPLC has been developed and validated for quantification of this drug in the solid dispersions, using PEG 6000 and Poloxamer 407 as polymers. The method was specific, linear in the range of $1.0-50.0 \mu \mathrm{g} \mathrm{mL} \mathrm{m}^{-1}(\mathrm{r}<0.99)$, precise $(\mathrm{CV}<5 \%$ for both inter- and intra-assays), accurate (maximum deviation of $\pm 13 \%$ ), and robust to the parameters evaluated. This method has proved to be simple and useful for ursolic acid determination in solid dispersions, enabling its determination in pharmaceutical dosage form.
\end{abstract}

Keywords: ursolic acid; quantitative analyses; HPLC.

\section{INTRODUÇÃO}

O ácido ursólico (AU), ácido 3 $\beta$-hidroxi-urs-12-en-28-óico (Figura 1), é um triterpeno $\left(\mathrm{C}_{30}\right)$ pentacíclico e normalmente surge associado ao seu isômero, o ácido oleanólico. ${ }^{1}$ Amplamente distribuído no reino vegetal, está presente em muitas ervas medicinais e outras plantas, incluindo ginseng (Panax ginseng), calêndula (Callendula officinalis), alecrim (Rosmarinus officinalis), melaleuca (Melaleuca leucadendron), maçã (Malus domestica), pera (Pyrus pyrifolia), ameixa (Prunus domestica), entre outras. ${ }^{1,2} \mathrm{O}$ AU tem uso consagrado na cosmética devido às suas ações antioxidante e renovadora celular. ${ }^{3}$ Nos últimos anos, o interesse em sua pesquisa tem aumentado, pois estudos indicam que o AU exibe variados efeitos farmacológicos, tais como atividade anti-inflamatória, hipoglicemiante, antioxidante, anti-hiperlipidêmica, imunomodulatória, antiangiogênica, antimutagênica, vasoprotetora, hepatoprotetora, antitumoral e tripanocida. ${ }^{4-13}$

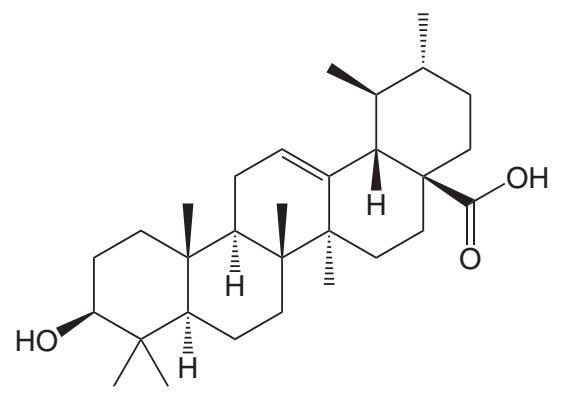

Figura 1. Estrutura química do ácido ursólico

Apesar do interesse crescente no ácido ursólico, sua baixa solubilidade em água é um fator limitante para sua biodisponibilidade. ${ }^{1}$ Diversas estratégias podem ser empregadas para melhorar a solubilidade de fármacos lipofílicos visando o aumento da biodisponibilidade. Dentre as mais eficazes, destacam-se as dispersões sólidas utilizando polímeros hidrofílicos, onde o princípio ativo se encontra molecularmente disperso ou em seu estado amorfo, sendo seu perfil de dissolução guiado pelas propriedades do carreador. ${ }^{14,15}$

*e-mail: jmarchet@usp.br
Sabe-se que os polímeros empregados desempenham um papel chave na melhora da dissolução de fármacos hidrofóbicos, o que torna sua seleção fundamental no desenvolvimento bem sucedido da dispersão sólida. Dentre estes polímeros, os mais frequentemente empregados como carreadores em dispersões sólidas são os polietilenoglicóis (PEGs) e as polivinilpirrolidonas (PVPs), ${ }^{14}$ dos quais o PVP-K30 já foi testado em dispersões sólidas de ácido ursólico, porém o aumento observado de solubilidade e dissolução foi pequeno com o uso deste carreador de $1^{a}$ geração. ${ }^{16,17}$ Recentemente, alguns fármacos tiveram um aumento considerável da solubilidade e da velocidade de dissolução com o uso de polímeros com propriedades tensoativas, de $3^{\text {a }}$ geração, como o poloxamer, que não somente aumenta a molhabilidade de fármacos hidrofóbicos, mas auxilia a dissolução destes pelo mecanismo da solubilização micelar. Além disso, o uso de tensoativos como carreadores pode conceder ao sistema a vantagem de inibição da recristalização do fármaco, um sério problema de instabilidade comum às dispersões sólidas..$^{14,18-20}$

Métodos analíticos têm sido desenvolvidos principalmente para avaliar e quantificar o ácido ursólico em plantas. ${ }^{21-23}$ Todavia, estes métodos envolvem etapas de extração na matriz vegetal e não seriam adequados para a análise em matrizes menos complexas, como as formas farmacêuticas. Diante disto, o desenvolvimento de um método analítico adequado para determinar o ácido ursólico em formas farmacêuticas, como dispersões sólidas com poloxamer, é bastante relevante.

O desenvolvimento de um método analítico implica na necessidade de garantir a qualidade dos resultados obtidos, assim como sua eficiência na rotina do laboratório. A validação do método é um processo contínuo que visa confirmar a confiabilidade e capacidade de desempenho do mesmo. ${ }^{24,25}$ Técnicas de separação como cromatografia gasosa (CG), cromatografia líquida de alta eficiência (CLAE) e eletroforese capilar (EC) têm destaque na Química Analítica pela capacidade de identificação de compostos por comparação com padrões, purificação de compostos separando-os de componentes de uma mistura seja ela biológica, farmacêutica ou alimentícia. ${ }^{24,26}$ Particularmente a CLAE é largamente empregada por possuir vantagens como curto tempo de preparo da amostra a ser analisada; dados qualitativos e quantitativos podem ser obtidos para amostras de polaridades variadas em uma só corrida de análise e, permite várias 
formas de detecção, podendo aumentar a especificidade e o conteúdo de informações por ensaio. ${ }^{27}$

O objetivo do presente estudo foi desenvolver e validar um método analítico empregando a cromatografia líquida de alta eficiência para a determinação do ácido ursólico em duas preparações de dispersões sólidas, utilizando PEG 6000 ou Poloxamer 407, o que permitirá comparação posterior entre estes dois polímeros como carreadores do fármaco em estudo.

\section{PARTE EXPERIMENTAL}

\section{Reagentes e soluções}

O ácido ursólico (AU) foi adquirido da Idealfarma (Brasil). O PEG 6000 e o Poloxamer 407 foram adquiridos da BASF (Alemanha). A acetonitrila e o metanol de grau cromatográfico foram adquiridos da J. T. Baker (EUA). A água foi obtida pelo Sistema Milli-Q Plus - Millipore ${ }^{\circledR} /$ Millipore Corporation (EUA).

\section{Métodos}

As dispersões sólidas foram preparadas pelo método da fusão, ${ }^{28}$ onde o AU foi adicionado ao carreador fundido (PEG 6000 ou Poloxamer 407) nas proporções fármaco/polímero 1:10 e 1:20 (p/p), a $300{ }^{\circ} \mathrm{C}$, com homogeneização contínua até a formação de dispersão homogênea, que, em seguida, foi solidificada por resfriamento em banho de gelo e pulverizada com o auxílio de gral e pistilo.

\section{Solução-padrão}

As soluções-padrão foram preparadas a partir de diluições de uma solução estoque do AU $100 \mu \mathrm{g} \mathrm{mL}^{-1}$ em acetonitrila.

\section{Desenvolvimento do método analítico}

Foi realizada varredura em espectrofotômetro UV-vis Femto 800XI (Brasil) na faixa de 190 a $400 \mathrm{~nm}$ a fim de determinar o comprimento de onda de absorção máxima do $\mathrm{AU}(\lambda)$.

\section{Condições cromatográficas}

As análises por CLAE foram realizadas em cromatógrafo a líquido Shimadzu (Japão) constituído por uma bomba modelo LC-10AD, detector UV-VIS modelo SPD-10AVP $(\lambda=203 \mathrm{~nm})$, injetor Rheodyne e integrador Chromatopac modelo C-R6A. A fase estacionária foi composta por uma coluna C18 Lichrospher $^{\circledR}$ Merk (d.i. 4,0 x 250 $\mathrm{mm} ; 5 \mu \mathrm{m}$ ) protegida por uma coluna de guarda (d.i. 4 x $4 \mathrm{~mm}) \mathrm{de}$ mesma composição. A fase móvel foi constituída de acetonitrila:água $(88: 12, v / v)$. As análises foram realizadas em temperatura ambiente controlada $\left(25,0 \pm 1,0{ }^{\circ} \mathrm{C}\right)$ utilizando uma vazão de $1,0 \mathrm{~mL} \mathrm{~min}{ }^{-1} \mathrm{e}$ volume de injeção de $20 \mu \mathrm{L}$. As amostras foram filtradas em membrana $0,45 \mu \mathrm{m}$ antes da injeção.

\section{Validação do método analítico}

Para validação do método foram avaliados os parâmetros de seletividade, linearidade, limites de detecção e quantificação, precisão, exatidão e robustez, seguindo as especificações da ANVISA ${ }^{29} \mathrm{e}$ recomendações do ICH Harmonised Tripartite Guideline. ${ }^{30}$

\section{Seletividade}

A seletividade foi determinada pela comparação dos resultados obtidos de amostras do AU contaminadas com quantidades apropriadas dos excipientes empregados nas dispersões sólidas, PEG 6000 e Poloxamer 407, e análise das dispersões sólidas sem o fármaco (placebos).

\section{$\underline{\text { Linearidade }}$}

A partir da solução estoque de $\mathrm{AU}\left(100,0 \mu \mathrm{g} \mathrm{mL}^{-1}\right)$ foram preparadas soluções em concentrações de 1,0; 5,0;10,0;25,0 e 50,0 $\mu \mathrm{g} \mathrm{mL}^{-1}$. As curvas analíticas foram construídas relacionando-se os valores da concentração em $\mu \mathrm{g} \mathrm{mL}^{-1}$, no eixo das abscissas, com os valores das áreas obtidas, no eixo das ordenadas. O intervalo linear foi calculado através da verificação da proporcionalidade entre a concentração e a resposta a partir do cálculo do coeficiente linear $(b)$, do coeficiente angular $(a)$ e do coeficiente de correlação (r) (r mínimo aceitável foi de 0,99). ${ }^{29}$ Foram realizadas análises estatísticas para avaliação da regressão linear.

\section{Precisão e exatidão}

A precisão foi avaliada através da repetibilidade e precisão intermediária. A exatidão foi avaliada intra e interensaio. Para avaliar estes parâmetros, foram utilizadas soluções de padrão de AU (1,0; 25,0 e 50,0 $\left.\mu \mathrm{g} \mathrm{mL}^{-1}\right)$. Para determinação da repetibilidade e da exatidão intraensaio, as análises de 10 replicatas foram realizadas em um mesmo dia; a exatidão interensaios e precisão intermediária foram avaliadas em triplicata, por 3 dias consecutivos. Os resultados da precisão do método foram apresentados através do coeficiente de variação $(\mathrm{CV})$, calculado pela razão do desvio padrão com a média dos valores obtidos.

Os resultados da exatidão intra e interensaios foram obtidos através da razão em porcentagem entre a diferença do valor obtido e o valor teórico, em relação ao valor teórico.

\section{Limites de detecção e quantificação}

O limite de detecção foi determinado através do sinal/ruído da linha de base através da relação entre o desvio padrão médio do intercepto com o eixo y com a inclinação da curva de calibração.

O limite de quantificação foi considerado como a menor concentração obtida com precisão e exatidão.

\section{Robustez}

Este parâmetro foi avaliado variando-se as seguintes condições analíticas: proporção dos solventes da fase móvel e a vazão empregada na análise.

\section{Determinação do teor de ácido ursólico em dispersões sólidas}

Para determinação do teor, amostras das dispersões sólidas (25 mg para dispersão sólida na proporção fármaco:carreador de 1:10 e 50 mg para dispersão sólida na proporção de 1:20) foram solubilizadas volumetricamente em $100 \mathrm{~mL}$ de acetonitrila e filtradas em membrana de $0,45 \mu \mathrm{m}$. Em seguida, alíquotas de 1,0 $\mathrm{mL}$ foram transferidas para balões volumétricos de $10 \mathrm{~mL}$ e o volume foi completado com acetonitrila. As amostras foram então analisadas por CLAE.

\section{RESULTADOS E DISCUSSÃO}

O desenvolvimento do método analítico para a determinação do AU iniciou-se com a definição do comprimento de onda a ser utilizado, através da varredura no espectro do ultravioleta, mostrando que o fármaco absorve fortemente em $203 \mathrm{~nm}$, o mesmo utilizado em trabalho prévio. ${ }^{31}$ Além disso, investigou-se a composição mais adequada da fase móvel, comparando-se os sistemas compostos por metanol:água e acetonitrila:água em diferentes proporções $(80: 20,85: 15,88: 12$ e 90:10 v/v). A fase móvel composta por acetonitrila:água $(88: 12 \mathrm{v} / \mathrm{v})$ mostrou ser a mais adequada, apresentando pico bem resolvido e tempo de retenção de $11 \mathrm{~min}$, como demonstrado na Figura 2. A fase móvel contendo metanol, por outro lado, revelou-se menos apropriada para a análise, pois o fármaco 
apresentou maior tempo de retenção, devido à maior polaridade do metanol em relação à acetonitrila. ${ }^{32}$ Além disso, a estabilização da linha de base foi mais demorada comparada à fase móvel composta por acetonitrila e água, apresentando bastante ruído. Este fato pode ser explicado pela maior absorvância do metanol $(205 \mathrm{~nm})$ com relação à acetonitrila $(190 \mathrm{~nm})$, e ao baixo $\lambda(203 \mathrm{~nm})$ utilizado para as análises. ${ }^{32}$ Ressalta-se a importância da seleção criteriosa da composição da fase móvel, pois idealmente se deseja que a corrida analítica seja a mais breve possível, usando mínimas quantidades de solvente orgânico, com consequências diretas para a redução do custo das análises.

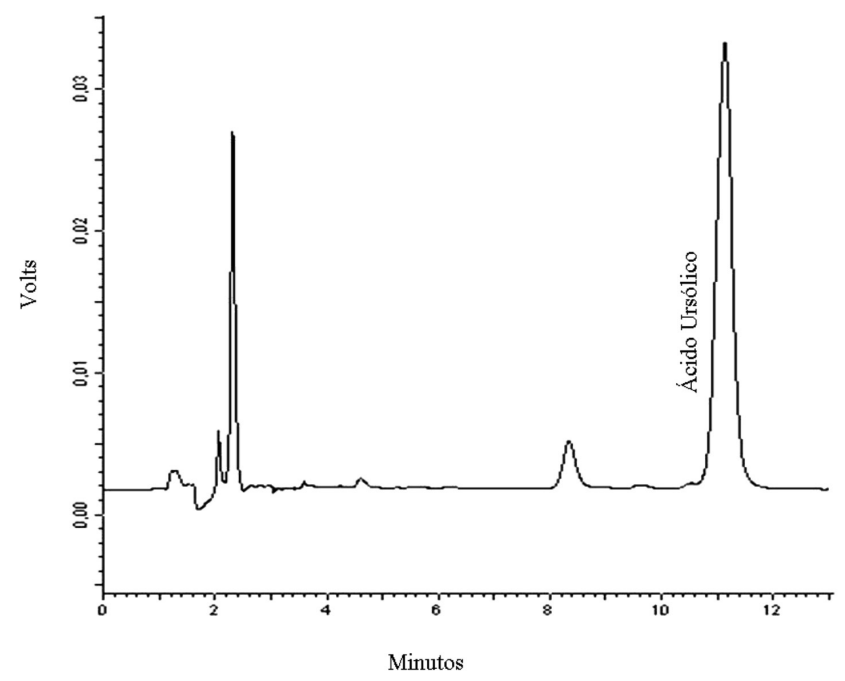

Figura 2. Cromatograma do ácido ursólico analisado por CLAE utilizando coluna C18, fase móvel composta por acetonitrila:água $(88: 12, v / v)$, vazão de 1,0 $\mathrm{mL} \mathrm{min}^{-1}$, em $203 \mathrm{~nm}$

\section{VALIDAÇÃO DO MÉTODO ANALÍTICO}

\section{Seletividade}

A seletividade é considerada a primeira etapa da validação analítica, pois se deve garantir que os componentes da formulação, como os excipientes, não interfiram na quantificação do fármaco. ${ }^{24}$ Os resultados obtidos pela análise das soluções de AU em acetonitrila contaminadas com os excipientes empregados nas dispersões sólidas, PEG 6000 e Poloxamer 407, e a análise das dispersões sólidas sem fármaco mostraram que estes polímeros não afetaram a quantificação do fármaco, porque não exibiram picos cromatográficos coincidentes com tempo de retenção característico do AU.

\section{Linearidade}

O método desenvolvido apresentou linearidade na faixa de

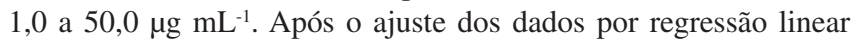
utilizando o método dos mínimos quadrados, os valores das áreas obtidas mostraram ser diretamente proporcionais à concentração de AU, no intervalo avaliado, [área $=13.300 x$ concentração $\left(\mu \mathrm{g} \mathrm{mL}^{-1}\right)$ $+272,25]$, com $r=0,9999$.

\section{Limites de detecção e quantificação}

O limite de quantificação, equivalente ao menor nível determinado com precisão e exatidão aceitáveis, correspondeu a $1,0 \mu \mathrm{g} \mathrm{mL} \mathrm{mL}^{-1}$. Concentrações abaixo desta foram analisadas e excluídas por não apresentarem exatidão. $\mathrm{O}$ limite de detecção foi de $0,34 \mu \mathrm{g} \mathrm{mL}^{-1}$.

\section{Precisão e exatidão}

Para a análise fidedigna do fármaco o método deve mostrar-se preciso, ou seja, apresentar pequena dispersão entre resultados de leitura de uma mesma concentração, e exato, representado pelo grau de concordância entre resultados individuais em um mesmo ensaio, ou ensaios independentes, em relação a um valor de referência aceito como verdadeiro. ${ }^{30}$ Os resultados apresentados na Tabela 1 evidenciaram que o método é preciso para ambas as determinações, repetibilidade, cujo CV máximo foi de 3,39\% para a menor concentração analisada, $1,0 \mu \mathrm{g} \mathrm{mL}^{-1}$, e interensaio, onde o $\mathrm{CV}$ máximo correspondeu a $4,49 \%$ para o limite de quantificação inferior a $1,0 \mu \mathrm{g} \mathrm{mL} \mathrm{mL}^{-1}$. A Tabela 2 mostra que o método desenvolvido é exato, já que os desvios com relação aos valores nominais mantiveram-se entre 0,28 e 3,04\%, para um mesmo dia de análise, e entre -4,11 e $0,20 \%$, para ensaios em dias diferentes.

Tabela 1. Precisão do método cromatográfico utilizado na análise do ácido ursólico

\begin{tabular}{lccc}
\hline Repetibilidade & & & \\
\hline Conc. teórica $\left(\mu \mathrm{g} \mathrm{mL}^{-1}\right)$ & 1,0 & 25,0 & 50,0 \\
$\mathrm{n}$ & 10 & 10 & 10 \\
$\mathrm{CV}(\%)$ & 3,39 & 0,70 & 1,16 \\
\hline Precisão intermediária & & & \\
\hline Conc. teórica $\left(\mu \mathrm{g} \mathrm{mL}^{-1}\right)$ & 1,0 & 25,0 & 50,0 \\
$\mathrm{n}$ & 3 & 3 & 3 \\
$\mathrm{CV}(\%)$ & 4,49 & 1,14 & 1,39 \\
\hline $\mathrm{Con}$
\end{tabular}

Conc.: concentração; n: número de replicatas.

Tabela 2. Exatidão do método cromatográfico utilizado na análise do ácido ursólico

\begin{tabular}{llll}
\hline Exatidão intraensaio & & & \\
\hline Conc. teórica $\left(\mu \mathrm{g} \mathrm{mL}^{-1}\right)$ & 1,00 & 25,00 & 50,00 \\
Conc. obtida $\left(\mu \mathrm{g} \mathrm{mL}^{-1}\right)$ & 1,03 & 24,69 & 50,14 \\
$\mathrm{n}$ & 10 & 10 & 10 \\
$\mathrm{E}(\%)$ & 3,04 & $-1,23$ & 0,28 \\
\hline Exatidão interensaio & & & \\
\hline Conc. teórica $\left(\mu \mathrm{g} \mathrm{mL}^{-1}\right)$ & 1,00 & 25,00 & 50,00 \\
Conc. obtida $\left(\mu \mathrm{g} \mathrm{m}^{-1}\right)$ & 0,96 & 24,68 & 50,10 \\
$\mathrm{n}$ & 3 & 3 & 3 \\
$\mathrm{E}(\%)$ & $-4,11$ & $-1,27$ & 0,20 \\
\hline
\end{tabular}

Conc.: concentração; n: número de replicatas.

\section{Robustez}

A robustez pode ser definida como a sensibilidade de um método frente a pequenas variações nas condições analíticas, tais como a proporção dos componentes da fase móvel e o fluxo empregado na análise. ${ }^{33}$ Os resultados expressos na Tabela 3 mostraram que o método analítico desenvolvido e validado se revelou robusto frente a alterações na vazão e composição da fase móvel, já que para todas as condições avaliadas o método manteve-se linear e exato (entre $-14,93 \%$ e $+10,50 \%)$.

\section{Determinação do teor de ácido ursólico em dispersões sólidas}

A partir do método validado, foram determinadas as concentrações de ácido ursólico em dispersões sólidas, que são formulações em forma de pó do fármaco disperso em um polímero hidrofílico, como o PEG 6000 e o Poloxamer 407, que são, respectivamente, um carreador clássico em dispersões sólidas e um tensoativo, correspondente à $3^{\mathrm{a}}$ 
Tabela 3. Robustez do método cromatográfico utilizado na análise do ácido ursólico

\begin{tabular}{|c|c|}
\hline \multicolumn{2}{|c|}{ Vazão $0,9\left(\mathrm{~mL} \mathrm{~min}^{-1}\right)$} \\
\hline \multicolumn{2}{|c|}{ Linearidade } \\
\hline $\mathrm{r}$ & 0,9999 \\
\hline \multicolumn{2}{|c|}{ Exatidão } \\
\hline Conc. teórica $\left(\mu \mathrm{g} \mathrm{mL} \mathrm{m}^{-1}\right)$ & $\mathrm{E}(\%)$ \\
\hline 1 & 2,35 \\
\hline 25 & 1,06 \\
\hline 50 & 0,33 \\
\hline \multicolumn{2}{|c|}{ Vazão $1,0\left(\mathrm{~mL} \mathrm{~min}^{-1}\right)$} \\
\hline \multicolumn{2}{|c|}{ Linearidade } \\
\hline $\mathrm{r}$ & 0,9999 \\
\hline \multicolumn{2}{|c|}{ Exatidão } \\
\hline Conc. teórica $\left(\mu \mathrm{g} \mathrm{mL} L^{-1}\right)$ & $\mathrm{E}(\%)$ \\
\hline 1 & 10,50 \\
\hline 25 & $-1,92$ \\
\hline 50 & 0,46 \\
\hline \multicolumn{2}{|c|}{ Fase Móvel (86,8:12,2 acetonitrila:água) } \\
\hline \multicolumn{2}{|c|}{ Linearidade } \\
\hline $\mathrm{r}$ & 0,9994 \\
\hline \multicolumn{2}{|c|}{ Exatidão } \\
\hline Conc. teórica $\left(\mu \mathrm{g} \mathrm{mL} \mathrm{m}^{-1}\right)$ & $\mathrm{E}(\%)$ \\
\hline 1 & $-14,93$ \\
\hline 25 & 4,64 \\
\hline 50 & $-1,07$ \\
\hline \multicolumn{2}{|c|}{ Fase Móvel (89,2:10,8 acetonitrila:água) } \\
\hline \multicolumn{2}{|c|}{ Linearidade } \\
\hline $\mathrm{r}$ & 0,9997 \\
\hline \multicolumn{2}{|c|}{ Exatidão } \\
\hline Conc. teórica $\left(\mu \mathrm{g} \mathrm{mL} \mathrm{m}^{-1}\right)$ & $\mathrm{E}(\%)$ \\
\hline 1 & 6,47 \\
\hline 25 & $-3,52$ \\
\hline 50 & 0,57 \\
\hline
\end{tabular}

geração deste tipo de formulação. ${ }^{14}$ Os dados na Tabela 4 mostraram que a recuperação do ácido ursólico a partir das formulações situou-se em intervalos compreendidos entre $97,87 \%$, para a formulação PEG 6000 , contendo $10 \%$ de fármaco, e $105,84 \%$, o valor mais elevado, para a formulação Poloxamer 407, contendo $20 \%$ de ácido ursólico, o que forneceu evidência da seletividade do método, já que os excipientes da formulação não afetaram a análise.

Os resultados obtidos mostraram que o método desenvolvido é útil para dosagem de ácido ursólico em dispersões sólidas preparadas com

Tabela 4. Determinação do teor de ácido ursólico em dispersões sólidas

\begin{tabular}{lcc}
\hline Formulação & Conc. obtida $\left(\mu \mathrm{gLL}^{-1}\right)$ & Teor $(\%)$ \\
\hline PEG 6000 (1:10) & 24,47 & 97,87 \\
PEG 6000 (1:20) & 26,21 & 104,85 \\
Poloxamer 407 (1:10) & 24,96 & 99,83 \\
Poloxamer 407 (1:20) & 24,46 & 105,84 \\
\hline
\end{tabular}

diferentes polímeros, como o PEG 6000 e Poloxamer 407. Estudos posteriores de caracterização físico-química e avaliação da solubilidade e perfil de dissolução dos sistemas desenvolvidos utilizarão o método desenvolvido neste trabalho, permitindo uma comparação entre os dois polímeros, quanto à eficácia como estratégia para o aumento da biodisponibilidade do ácido ursólico.

\section{CONCLUSÃO}

O método proposto para análise do ácido ursólico utilizando a técnica de CLAE foi validado e mostrou-se seletivo, linear na

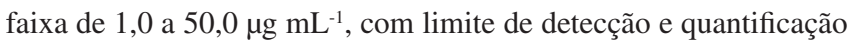
equivalentes a 0,34 e $1,0 \mu \mathrm{g} \mathrm{mL}^{-1}$, respectivamente, preciso, exato e robusto frente a variações de composição e fluxo de fase móvel, e provou ser conveniente e confiável, podendo ser aplicável ao controle de qualidade de formulações farmacêuticas baseadas em dispersões sólidas de PEG 6000 e Poloxamer 407.

\section{AGRADECIMENTOS}

Ao suporte financeiro da CAPES, FAPESP e à Faculdade de Ciências Farmacêuticas de Ribeirão Preto, Universidade de São Paulo (FCFRP-USP).

\section{REFERÊNCIAS}

1. Liu, J.; J. Ethnopharmacol. 2005, 100, 92.

2. Prasad, S.; Yadav, V. R.; Kannappan, R.; Aggarwal, B. B.; J. Biol. Chem. 2011, 286, 5546.

3. Haida, K. S.; Baron, A.; Silva, F. J.; Arceles, M. L.; Fernandes, A.; Andreazza, A. P.; Costa, J. H. B.; Rev. Saud. Pesq. 2011, 4, 61.

4. Baricevic, D.; Sosa, S.; Della Loggia, R.; Tubaro, A.; Simonovska, B.; Krasna, A.; Zupancic, A.; J. Ethnopharmacol. 2001, 75, 125.

5. Lee, J.; Yee, S. T.; Kim, J. J.; Choi, M. S.; Kwon, E. Y.; Seo, K. I.; Lee, M. K.; Chem. Biol. Interact. 2010, 108, 635.

6. Somova, L. A.; Nadar, A.; Rammanan, P.; Shode, F. O.; Phytomedicine $\mathbf{2 0 0 3}, 10,115$

7. Raphael, T. J.; Kuttan, G.; Phytomedicine 2003, 10, 483.

8. Cárdenas, C.; Quesada, A. R.; Media, M. A.; Biochem. Biophys. Res. Commun. 2004, 320, 402.

9. Resende, F. A.; Barcala, C. A. M. A.; Faria, M. C. S.; Kato, F. H.; Cunha, W. R.; Tavares, D. C.; Life Sci. 2006, 79, 1268.

10. Crespo, F. A.; Galicia, J. V.; Molina, R. V.; Guerrero, J. J. R.; Vázquez, G. N.; Soto, S. E.; Life Sci. 2006, 79, 1062.

11. Saravanan, R.; Viswanathan, P.; Pugalendi, K.V.; Life Sci. 2006, 78, 713.

12. Vechia, L. D.; Gnoatto, S. C. B.; Gosmann, G.; Quim. Nova 2009, 32, 1245.

13. Ferreira, D. S.; Esperandim, V. R.; Toldo, M. P. A.; Saraiva, J.; Cunha, W. R.; Albuquerque, S.; Parasitol. Res. 2010, 106, 985.

14. Vasconcelos, T.; Sarmento, B.; Costa, P.; Drug Discov. Today 2007, 12, 1068.

15. Craig, Q. M. D.; Int. J. Pharm. 2002, 231, 31.

16. Wu, C.; Shengquin, Z.; Lishizhen Med. Mat. Med. Res. 2007, 18, 2075.

17. Wu, C.; Shengquin, Z.; Jiangxi Norm. Univ. 2006, 30, 81.

18. Goddeeris, C.; van den Mooter, G.; Euro. J. Pharm. Sci. 2008, 35, 104.

19. Nepal, P. R.; Han, H. K.; Choi, H. K.; Int. J. Pharm. 2010, 383, 147.

20. Ali, W.; Williams, A. C.; Rawlinson, C. F.; Int. J. Pharm. 2010, 391, 162.

21. Li, G.; Zhang, X.; You, J.; Song, C.; Sun, Z.; Xia, L.; Suo, Y.; Anal. Chim. Acta 2011, 688, 208.

22. Kontogiani, V. G.; Exarchou, V.; Troganis, A.; Gerothanassis, I. P.; Anal. Chim. Acta 2009, 635, 188.

23. Olszewska, M.; Acta Chromatographica 2008, 20, 643. 
24. Ribani, M.; Bottoli, C. B. G.; Collins, C. H.; Jardim, I. C. S. F.; Melo, L. F. C.; Quim. Nova 2004, 27, 771.

25. Brito, N. M.; Amarante-Junior, O. P.; Polese, L.; Ribeiro, M. L.; Pesticidas: R. Ecotoxicol. e Meio Ambiente. 2003, 13, 129.

26. Degani, A. L. G.; Cass, Q. B.; Vieira, P. C.; Química Nova na Escola 1997, $\mathrm{n}^{\circ} 7,21$.

27. Karcher, B. D.; Davies, M. L.; Delaney, E. J.; Venit, J. J.; J. Assoc. Lab. Automation 2005, 10, 381.

28. Bley, H.; Fussnegger, B.; Bodmeir, R.; Int. J. Pharm. 2010, 390, 165.

29. Brasil, Agência Nacional de Vigilância Sanitária (ANVISA); Resolução $n^{\circ} R E 899$, de 29 de maio de 2003.
30. ICH - International Conference on Harmonisation of Technical Requirements for Registration of Pharmaceuticals for Human Use; ICH Harmonised Tripartite Guideline. Validation of analytical procedures: text and methodology Q2(R1), 2005.

31. Gnoatto, S. C. B.; Schenkel, E. P. ; Bassani, V. L.; J. Braz. Chem. Soc, 2005, 16, 723.

32. Snyder, L. R.; Kirkland, J. J.; Dolan, J. W.; Introduction to modern liquid chromatography, $3^{\text {rd }}$ ed., John Wiley \& Sons, Inc.: Hoboken, 2010, p. 960.

33. Instituto Nacional de Metrologia, Normalização e Qualidade Industrial (INMETRO); Orientações sobre Validação de Métodos de Ensaios Químicos, DOQ-CGCRE-008, 2003. 